\title{
El uso de redes sociales en migrantes Colombianos en Chile*
}

\author{
Use of Social Networks by Colombian Migrants in Chile \\ $\mathrm{O}$ uso de redes sociais em migrantes colombianos no Chile
}

Isabel Pavez Andonaegui ${ }^{\text {a }}$

Universidad de los Andes, Chile

DOI: https://doi.org/10.11144/Javeriana.syp39-76.ursm

mipavez@uandes.cl

ORCID: https://orcid.org/0000-0002-8367-4793

Recibido: 20 Marzo 2019

Cecilia Claro

Universidad de los Andes, Chile

ORCID: https://orcid.org/0000-0002-3428-0616

Aceptado: 27 Noviembre 2019

Publicado: 30 Junio 2020

\author{
Julián Andrés Burgos Suárez \\ Universidad de Manizales, Colombia \\ ORCID: https://orcid.org/0000-0001-7302-2866
}

\section{Resumen:}

Este artículo explora la forma en que las redes sociales permiten a los migrantes y sus familias transnacionales, controlar cuándo y cómo comunicarse, tanto para estar en contacto en tiempo real con sus comunidades de origen, como para mantener prácticas y tradiciones, logrando una copresencialidad (Baldassar, 2016). Se empleó una metodología cualitativa que incluyó entrevistas diádicas a veinte participantes de diferentes familias colombianas, que experimentaron la migración de uno de sus miembros a Chile. Entre los resultados, se destaca que WhatsApp es la red social más utilizada debido a la variedad de posibilidades para conectarse de forma sincrónica y asincrónica. Los participantes reportaron un aumento en la sensación de separación y distancia a pesar de la cercanía digital. La evidencia también indica el esfuerzo por parte de los migrantes, de prolongar sus roles de madre, hermano o hijo a través del uso de redes sociales.

Palabras clave: migración, internet, redes sociales, roles familiares, copresencialidad.

\section{Abstract:}

This paper explores how social networks allow that migrants and their transnational families may control when and how communicate in order to keep in contact in real time with their homeland communities, as well as preserving their traditions and practices. This way, they keep a copresentiality (Baldassar, 2016). This work is conducted under a qualitative methodology including dyadic interviews to twenty subjects from different Colombian families who experienced that one of their family members went to Chile. The results show noticeably that WhatsApp is the most frequently used social network due to its assorted features for getting connected both synchronically and diachronically. The subjects reported an increase in the feeling of distancing and separation despite the digitally-enabled closeness. The evidence also shows some efforts by the migrants to maintain their roles as a mother, a brother or a son thanks to the use of social networks.

Keywords: migration, Internet, social networks, family roles, copresentiality.

\section{Resumo:}

Este artigo explora a forma em que as redes sociais permitem que os migrantes e suas famílias transnacionais controlem quando e como se comunicar, tanto para estar em contato em tempo real com as comunidades de origem quanto para manter práticas e tradições, obtendo a copresencia (Baldassar, 2016). Foi usada metodologia qualitativa que incluiu entrevistas didáticas a vinte participantes de diferentes famílias colombianas, que vivenciaram a migração de um dos seus membros para o Chile. Dentre os resultados destaca-se que WhatsApp é a rede social mais utilizada devido à variedade de possibilidades de conexão de forma síncrona e assíncrona. Os participantes relataram um aumento na sensação de separação e distância apesar da proximidade digital. As evidências mesmo indicam esforço por parte dos migrantes, em prolongar seus papeis de mãe, irmão ou filho através do uso de redes sociais.

Palavras-chave: migração, internet, redes sociais, papeis familiares, copresencia.

Notas de autor

\footnotetext{
${ }^{\mathrm{a}}$ Autor de correspondencia. Correo electrónico: mipavez@uandes.cl
} 


\section{Introducción}

La movilidad y acceso que permiten los avances tecnológicos, han apoyado las poblaciones migrantes, transformando el proceso de inserción en una sociedad extranjera (Benitez, 2006; Kim et al., 2013; Pavez, 2014). Aunque hubo un momento en la historia en que los migrantes se vieron obligados a "abandonar, olvidar o negar sus vínculos con el hogar" (Schiller et al., 1995, p. 51) las tecnologías de comunicación e información (TIC) transformaron completamente la manera en que se vinculan con sus seres queridos a pesar de la separación geográfica (Baldassar, 2016), dando lugar al migrante conectado (Diminescu, 2008). Esto se da, porque los desarrollos tecnológicos les ofrecen el potencial de controlar cuándo, cómo se preocupan y ocupan de esta distancia, dándoles la posibilidad de conectarse en tiempo real con sus comunidades de origen lo que les permite mantener prácticas y tradiciones (Baldassar, 2016; Wilding, 2006). En otras palabras, "hacer familia desde lejos" (Peñaranda et al., 2011, p. 27) creando espacios que permiten mantener los vínculos entre los miembros de familias transnacionales, donde las TIC desempeñan un papel crucial en el desarrollo de redes y el refuerzo de las configuraciones sociales (Vertovec, 2009). Por ello, en el paisaje migratorio las tecnologías se convierten en algo más que canales de comunicación, pues son elementos claves para la selección del país donde se migrará, y más aún, en la extensión del tiempo dadas las facilidades comunicativas con la comunidad de origen (Madianou, 2014, 2016).

Sin embargo, para abordar el papel de las tecnologías en el ámbito transnacional, es necesario evidenciar las complejidades de los contextos de los migrantes, su vida cotidiana, y la forma en que estas ubicaciones geográficas dan paso a conflictuados sentidos de pertenencia (Georgiou, 2006, 2013; Bailey, 2007). En otras palabras, implica reconocer que sus identidades y códigos culturales, así como su comunidad de referencia, ya no hacen parte de su nuevo entorno (Hiller y Franz, 2004). Se trata de un escenario complejo porque, aunque este fenómeno ilumina las ambigüedades y tensiones en torno a las conexiones emocionales de "aquí" y "allá" (Boccagni y Baldassar, 2015, p. 74), los avances tecnológicos no permiten enfocar las comunicaciones y relaciones de los migrantes en un espacio binario, compuesto por la comunidad de acogida y comunidad de origen. La existencia de espacios translocales (Georgiou, 2006; Witteborn, 2012) que desafían los límites geopolíticos o sociopolíticos, da lugar a un entorno digital con multitud de conexiones (Burrell y Anderson, 2008). Hoy en día estas fronteras son límites confusos, pues aspectos como la movilidad y la conectividad dan cuenta de un espacio que va más allá de lo geográfico, complejizando las texturas de los espacios comunicativos.

Estos avances y nuevas prácticas digitales les permiten a los migrantes satisfacer una variedad de necesidades tales como, mantener hábitos y rituales que alimentan sus identidades culturales (Georgiou, 2005; Gillespie, 1995), dando lugar a espacios de inclusión, pertenencia o segregación (Bailey, 2007). De esta manera, su establecimiento en una nueva sociedad se ha convertido en un producto del desempeño tecnológico que les permite vivir una vida transnacional (Vertovec, 2009). Por esta razón, la literatura se refiere a espacios híbridos resultantes de un consumo globalizado (Bhabha, 1996; Bailey et al.,2007; Christiansen, 2004). Estos espacios muestran diferentes identificaciones que se traducen en formas dinámicas en las que se utiliza la tecnología, así como las redes sociales (Brüggemann y Wessler, 2014; Karim, 1998; Lutz, 2011). Experiencias que no son desterritorializadas porque se nutren continuamente tanto en el online, como en el offline (Miller y Slater, 2000; Madianou y Miller, 2012), reforzando el argumento de que la vida cotidiana es un componente esencial al analizar el papel de las tecnologías en el panorama de la migración.

Es por ello, que el foco de este artículo está puesto en cómo el uso de TIC en los migrantes, principalmente el de redes sociales, se relaciona con sus procesos de mantención de vínculos con sus comunidades de origen. Sobre la base de entrevistas en profundidad a veinte inmigrantes colombianos en Chile y a sus familiares en sus ciudades de origen, la pregunta de investigación que explora este documento es: ¿Cuál 
es el papel de la tecnología, en particular las redes sociales, en el día a día de las familias separadas por migración? Teóricamente, este enfoque permite dar respuestas de cómo internet cobra significado en un contexto migratorio en una población que es de alto interés debido al cambio cultural que se ha registrado, tanto en Latinoamérica como en Chile, desde hace una década (Cano y Sofia, 2009; Thayer et al., 2013; Martínez y Orrego, 2016). Saber a cuáles medios y tecnologías acceden y por qué, entendido como el contexto simbólico de su apropiación (Courtois et al., 2012; Hartmann, 2006) entrega elementos que permiten explorar necesidades emocionales desencadenadas por las condiciones de migración y estrategias para mantener sus vínculos familiares.

\section{Construyendo redes digitales}

En el pasado, los cibercafés representaban un espacio esencial para los migrantes porque eran el lugar de acceso a internet donde se conectaban, con sus países de origen y miembros de su familia. No obstante, el desarrollo de las comunicaciones móviles dejó atrás estos populares espacios (Banco de Desarrollo de América Latina, 2013) transformando los tiempos y las dinámicas de las comunicaciones en la familia transnacional. A pesar de este cambio en la experiencia migratoria, la investigación sobre el tema desde el punto de vista de las emociones y las relaciones de los migrantes continúa siendo poco estudiada (Boccagni y Baldassar, 2015), en particular en las poblaciones migrantes sur-sur. En este sentido, América Latina es un caso de estudio interesante puesto que es la región que más horas utiliza en redes sociales, superando a Europa y Norteamérica (Torres y Aguilar, 2018)

Este tema es relevante porque la evidencia indica que los usos tecnológicos y el consumo de medios, tanto en su diversidad como complemento-, apuntan a prácticas que ayudan a los migrantes a sobrellevar la nostalgia (Feng y Ekiaka, 2011; Pavez, 2014). Es aquí donde los smartphones, y en particular las redes sociales, adquieren un rol clave puesto que permiten acceder a una comunicación instantánea, móvil y enriquecida, la cual tiene un papel en la continuidad así como en la creación de nuevas dinámicas entre las familias separadas por la migración. Esto influye en cómo los migrantes se adaptan a la comunidad de acogida, pero también en cómo integran a sus seres queridos en su vida cotidiana (Doveling et al., 2018). Este cambio permite plantearse preguntas sobre cómo la familia del migrante se adapta a la ausencia de un miembro y cómo lo incorporan (o no) a sus asuntos habituales. Por ejemplo, King-O'Riain (2015) estudió cómo las familias transnacionales que viven en Irlanda utilizan Skype para interactuar con sus seres queridos y transmitir afectividad. Con una muestra de 36 entrevistas, entre los hallazgos, destaca que "las familias transnacionales no están desconectadas emocionalmente, sino que están integradas en localidades facilitadas por el uso creciente de tecnologías como Skype" (p. 271). Estas video-llamadas que emulan conversaciones reales, les permite desplegar sus sentimientos y conectarse emocionalmente con una rutina diaria, fueron quizás el primer paso en este cambio que se consolida con la llegada de aplicaciones para teléfonos inteligentes, más accesibles y menos engorrosas que gozan de gran popularidad en Latinoamérica. Por ejemplo, la penetración de las redes sociales llegó en 2018 a 63\% en promedio, alcanzando Chile un 77\% de uso, mientras que en Colombia un 63\% (Hootsuit, 2018).

Esta amplia adopción de Internet y redes sociales han sido claves en el proceso porque abren la puerta a la omnipresencia de las plataformas de comunicación móviles, instantáneas y enriquecidas, convirtiéndose en un hito para los vínculos de las redes de migrantes (Dekker et al., 2016). Estos desarrollos tecnológicos han proporcionado una nueva salida para la unión familiar debido a la proximidad digital y los espacios de intimidad, lo que les permite desempeñar en cierta medida sus roles en el ámbito digital. No obstante, es erróneo argumentar que la familia mantiene los mismos lazos como producto de estas prácticas digitales. Por el contrario, hay nuevos significados y formas de abordar la vida social y familiar, que cambian los códigos temporales y espaciales que dan paso a nuevas narrativas (Bell y Erdal, 2015). Por ejemplo, los sentidos de 
pertenencia y la concepción de la familia cambian con el tiempo en el extranjero, obligando a los migrantes a adaptarse a una "familia imaginada" (Vermot, 2015, p.145). Un ejemplo de esto es la investigación de Katz y González (2016), cuyo resultado indica que las TIC ayudan a mantener una intimidad virtual con familiares, obtener apoyo emocional y participar en la atención transnacional, brindando apoyo a los esfuerzos de mantener la continuidad familiar. Además, estos desarrollos han ayudado a lidiar con temas como la cercanía, redefiniendo las relaciones transnacionales y asegurándose de llevar con ellos, de una forma virtual, a los que dejaron atrás (Francisco, 2015).

\section{Migración y smartphones: ¿siempre disponible?}

Aunque el uso e inserción de teléfonos inteligentes entre los migrantes es de gran relevancia, una de las preguntas que persiste es sobre la naturaleza y el entorno del intercambio de comunicación entre los miembros de la familia, cuáles medios particulares se utilizan para la comunicación transnacional (Dekker et al., 2016). Algunos lo han descrito como un artefacto portátil que permite a las familias adaptarse a los nuevos patrones de comunicación para incorporar al migrante en las rutinas diarias (Ramírez, 2014. Por ejemplo, Chib et al. (2014) estudiaron el papel del uso de teléfonos móviles en el tratamiento de las tensiones y ambivalencias derivadas de la maternidad transnacional entre las familias indonesias y filipinas. Los hallazgos indican que prefirieron los teléfonos inteligentes porque "desempeñaron un papel importante en el fortalecimiento de las uniones de padres que se centraron en la crianza de los hijos” (p. 86), pues ayudó a aumentar el contacto y la supervisión como una forma para aliviar el miedo, su principal emoción. En otra investigación similar, Madianou (2014) señala que los teléfonos inteligentes se consideran instrumentos que brindan un canal de comunicación para expresar emociones y también administran las relaciones familiares con los miembros que permanecen en Filipinas. En su proyecto de larga data (2007-2011), fue testigo de la evolución de los teléfonos inteligentes, en particular de la ubicuidad en lo que respecta a la conexión familiar, que se destacó por la idea de "estar en línea" o "estar siempre" como su único estado (p. 678).

Estos aspectos dan lugar a la idea de copresencia debido al "uso intensivo de la comunicación basada en Internet, los teléfonos móviles y las redes sociales [que] pueden contribuir a fortalecer los lazos e intensificar la circulación de diversos recursos (culturales, emocionales, económicos y sociales) dentro de las familias transnacionales" (Baldassar, 2016, p. 134). Entonces, la familia está unida simbólicamente en el ámbito digital, brindando apoyo y compañía a través del acceso a la voz y la imagen (Peñaranda, 2010; Rodríguez, 2011). Según Peñaranda (2010), es crucial poder conectar e interpretar más allá de las palabras y, además, disminuir la ansiedad producida por la separación. Por ejemplo, Tuñón y Mena (2018) analizan cómo el uso de las redes sociales y las TIC ayudan a las familias mexicanas fracturadas a lidiar con los sentidos del lugar y la pertenencia. Argumentan que la tecnología proporciona un escenario virtual para las familias que les permite disminuir la sensación de distancia mediante una presencia virtual y un contacto diario a través de mensajes de chat y el uso de las redes sociales. Estos hallazgos son consistentes con otra evidencia que muestra por ejemplo que, las familias y parejas utilizan los nuevos medios de manera creativa, como dejar la webcam encendida durante horas, lo que da la idea de estar allí (Madianou, 2014).

Estos espacios online y offline interactúan constantemente, cambiando las rutinas de comunicación, reconfigurando hábitos y rituales, con una amplia gama de escenarios tanto comunicacionales como emocionales. Tal y como sostienen Nedelcu y Wyss (2016): "generan efectos ambivalentes que van desde el bienestar recíproco inmediato y el confort emocional a nuevas expectativas de solidaridad, tensiones familiares y limitaciones" (p. 202). Los autores argumentan que esta co-presencia tecnológica permite que las familias continúen sus relaciones con éxito. En parte, porque el tiempo de respuesta al igual que la velocidad en que se llevan a cabo, facilita el intercambio comunicacional y los espacios para compartir eventos o noticias, incluso en tiempo real, fortaleciendo el vínculo (Peñaranda, 2010), y proporcionando nuevos rituales de comunicación (Brüggemann y Wessler, 2014). Estas prácticas y su regularidad son la base de los lazos 
emocionales entre las familias transnacionales que cambian y enriquecen su vida cotidiana, principalmente debido a la incorporación de medios tales como videos (Ramírez, 2014). Por ejemplo, Toral (2017) estudió las relaciones familiares a través del análisis del acceso, uso, frecuencia y tipo de información que los migrantes ecuatorianos junto con sus familiares obtienen de las TIC como herramientas alternativas de comunicación. Los resultados indican que la mayoría de los migrantes tienen comunicación diaria o al menos una vez a la semana con sus familias la cual figura dentro de sus prioridades en conjunto con actividades como el envío de remesas.

En un escenario migratorio, la comunicación en muchos casos se relaciona con el apoyo emocional, el intercambio de información, la resolución de problemas o conflictos así como la participación o la presencia del migrante en fechas especiales o de crisis de un miembro en el país de origen (Contreras, 2014). Sin embargo, estas prácticas tienen repercusiones en la integración a la comunidad de acogida. Por ejemplo, un estudio de Verdery et al. (2017) sobre cómo los migrantes se conectaban con sus comunidades de origen, encontraron que las llamadas frecuentes, los correos electrónicos o incluso las videoconferencias domésticas sustituyen en parte a las redes de amistad locales en el destino. También tiene efectos en la adopción tecnológica en la familia que permanece en el país de origen, puesto que estudios han dado cuenta que aumenta el interés por las TIC, y que las familias transnacionales son más propensas a adquirir dispositivos tecnológicos como computadoras y teléfonos inteligentes en comparación a familias no transnacionales (Pearce y Rice, 2013). Los investigadores afirman que,

el uso de las TIC para contrarrestar la fragmentación física de las familias crea una integración virtual de los miembros de la familia ausentes, lo que a su vez puede generar un efecto psicológico que ayuda a los 'dejados atrás' a sobrellevar la distancia. (p. 2149)

Esto se relaciona con lo que Bailey (2007) denomina un espacio de inclusión, permitiendo una "presencia continua a pesar de la distancia" (p. 572), cambiando por completo la forma en que las personas se adaptan a esta realidad fragmentada, pero conectada. Aunque ciertamente internet no tiene el poder de cambiar las relaciones, puede hacerlas posibles a través de canales de comunicación donde se pueden construir puentes para mantener las prácticas y tradiciones encontrando a través del espacio digital la cercanía que necesitan en un contexto vulnerable, pero también aumentando la sensación de distancia (Wilding, 2006).

\section{Colombianos en Chile como caso de estudio}

Chile es uno de los países Latinoamericanos que más migrantes ha recibido en la última década. Las estadísticas dan cuenta de un aumento anual desde el 2010 al 2015 de un 4,9\% (Cepal y Organización Internacional del Trabajo, 2017), llegando a 1.200 .000 al 31 de diciembre de 2017, que es equivalente al 6,1\% de la población del país (Departamento de Extranjería y Migración, s.f.). Históricamente, en América del Sur, Colombia ha sido el país que ha generado los mayores flujos de emigración y Chile se ha convertido en uno de los principales destinos. En 2019 se estimaba que residían en en Chile alrededor de 146.000 colombianos, siendo el cuarto país con mayores migraciones después de Venezuela, Perú y Haití (Departamento de Extranjería y Migración [DEM], 2019) Muchas causas influyen en la decisión de migrar. La principal razón es económica (82,7\%), específicamente la búsqueda de oportunidades de empleo; seguido de motivos personales como el matrimonio o la reunificación familiar; la tercera razón es la formación educativa; y, en menor medida, la falta de seguridad en Colombia (Taylor, 2014).

Si bien esta realidad ha obligado a discutir en la necesidad de la generación políticas públicas integrales, orientadas a avanzar en procesos de acogida, bienestar y cohesión social (Ávalos, 2012; Thayer et al., 2013; Rojas y Silva, 2016), a nivel nacional se da cuenta de un escenario complejo para el migrante. Por ejemplo, se ha caracterizado a Chile como una comunidad de acogida hostil, reportando truncadas experiencias de integración ligadas a prejuicios, vulnerabilidad y exclusión (Silva y Lufin, 2013; Méndez y Cárdenas, 2012) 
con efectos en depresión, ansiedad y estrés por parte de quienes buscan ser parte de la sociedad chilena (Yañez y Cárdenas, 2010).

Sin embargo, los escasos estudios del tema en migrantes en Chile dan cuenta que las tecnologías, en especial internet, permiten mantener redes transnacionales, alimentando su identidad y sentido de pertenencia tal como han dado cuenta otros estudios (Brinkerhoff, 2009; Chen, 2010). Entonces internet ayudaría a los migrantes a reforzar prácticas anteriores y acceder a códigos culturales que les son cercanos o comunes (Vertovec, 2009). De esta manera se posicionan digitalmente en su comunidad de referencia, sosteniendo vínculos con sus países de origen, y manteniendo prácticas y hábitos de consumo a pesar de la distancia (Burrell y Anderson, 2008; de Leeuw y Rydin, 2007; Greschke, 2012). Investigaciones desde el área de estudios Latinoamericanos han abordado la percepción de integración de colombianos en Antofagasta, concluyendo que su integración es compleja y que mantienen vínculos con el país de origen (Taylor, 2014). En tanto desde la psicología se ha explorado el bienestar psicológico y estrategias de integración en Afrocolombianos en el norte de Chile, donde el consumo cultural del migrante juega un papel relevante (Silva et al., 2016), lo cual permite plantear interesantes interrogantes ligadas al uso y consumo de redes sociales así como sus prácticas de comunicación con familiares en su país de origen.

\section{Metodología}

Esta investigación tiene como objetivo comprender cómo la tecnología, en particular las redes sociales, desempeñan un papel en el día a día de las familias separadas por la migración. Para lograrlo, se analiza cómo Internet les permite conectarse, pero también crear nuevos patrones de comunicación para mantener sus roles familiares ya sea de madre, hijo o hermano. La muestra fue compuesta por colombianos, grupo que tiene la cuarta fuerza migratoria en Chile con un 14,9\% de inmigrantes (Libertad y Desarrollo, 2019). También se seleccionaron migrantes que llegaron solos, es decir, sin alguien de su grupo familiar. Si bien se incorporó una amplia variedad de edades (entre los 20 - 55 años), y tiempo en Chile, todos responden a un segmento vulnerable con salarios más bajos que el promedio en el país anfitrión (aproximadamente 268US\$). Esta decisión responde a que la necesidad económica es una de las principales razones para migrar a Chile (Taylor, 2014). Más aún, porque es una condición que da cuenta de la vulnerabilidad en la que se encuentran, sin la libertad de viajar de forma permanente a ver a sus familiares, condición que permite explorar de forma más acentuada el rol de la tecnología en un escenario migratorio complejo desde el punto de vista de redes de apoyo (ver tabla 1 ).

TABLA 1.

\begin{tabular}{|c|c|c|c|c|c|c|}
\hline & Edad & Chile & Colombia & Edad & Ocupación & Relación familiar \\
\hline Rocio & 24 & No reportada & Francisca & 20 & Estudiante & Hermanas \\
\hline Cecilia & 23 & Limpieza & Magdalena & 45 & $\begin{array}{l}\text { Dueña de } \\
\text { casa }\end{array}$ & Hija - Madre \\
\hline Elisa & 21 & Limpieza & Carmen & 47 & Comerciante & Hija - Madre \\
\hline Nicole & 27 & $\begin{array}{l}\text { Ayudante de } \\
\text { cocina }\end{array}$ & María & 52 & $\begin{array}{l}\text { Dueña de } \\
\text { casa }\end{array}$ & Hija - Madre \\
\hline Julián & 22 & No reportada & Beatriz & 60 & Comerciante & Hijo - Madre \\
\hline José & 35 & $\begin{array}{l}\text { Ayudante de } \\
\text { cocina }\end{array}$ & Fernanda & 55 & $\begin{array}{c}\text { Dueña de } \\
\text { casa }\end{array}$ & Hijo - Madre \\
\hline Luz & 45 & $\begin{array}{c}\text { Administradora } \\
\text { de local }\end{array}$ & Manuela & 18 & Estudiante & Madre - Hija \\
\hline Ana & 48 & Vendedora & Isabel & 21 & Limpieza & Madre- Hija \\
\hline Patricia & 47 & Comerciante & $\begin{array}{c}\text { Juan } \\
\text { Ignacio }\end{array}$ & 23 & Estudiante & Madre - Hijo \\
\hline Jackie & 35 & $\begin{array}{l}\text { Ayudante } \\
\text { negocio }\end{array}$ & Francisco & 37 & Comerciante & Hermanos \\
\hline
\end{tabular}

Fuente: elaboración propia

En cuanto al reclutamiento, se inició invitando a los participantes en Santiago de Chile. Fueron contactados a través de una estrategia de bola de nieve en lugares comúnmente visitados por colombianos, como mercados y oficinas de migración. Cuando aceptaron participar, se llevó a cabo una breve encuesta de pertinencia para 
confirmar su estado como migrantes y usuarios de Internet, y también que tienen a un familiar cercano en su país de origen. Luego, se invitó al miembro de la familia en Colombia, quien también respondió la misma encuesta para validar la información proporcionada por el participante en Chile y su disposición a participar en el estudio. Posteriormente, se entrevistó a ambas partes, lo que fue facilitado por tener un investigador en Colombia y dos en Chile.

La estrategia metodológica para un tema tan sensible fue cualitativa, para poder contextualizar las experiencias de los participantes y darles voz, permitiendo surgir su comprensión del papel en la tecnología (Bryman, 2012; Esterberg, 2002), para lograr además un "entendimiento de textura fina” (Gaskell, 2000, p. 39). Por otro lado, las entrevistas diádicas, son una técnica que permite la interacción entre los participantes y brindaron la ventaja de explorar el tema desde ambos extremos (Morgan, Ataie et al., 2013; Morgan, Eliot et al. 2016). Sin embargo, la desventaja de los métodos cualitativos es la falta de representatividad y muestra una imagen del momento, no necesariamente una tendencia constante.

Las entrevistas se realizaron entre julio y octubre de 2018 en Chile y Colombia, luego se transcribieron. Para el análisis, se realizó una codificación temática siguiendo a Boyatzis (1998). Esto corresponde a la organización de segmentos temáticos que se extienden a través de las entrevistas y se pueden etiquetar mediante la creación de códigos, que ayudan a mapear temas, hallazgos detallados y cuestiones emergentes (Fielding y Thomas, 2008). Los principales temas de análisis fueron guiados por la revisión de la literatura y cubrieron aspectos como (1) experiencias de migración, (2) lazos familiares, (3) acceso y uso de Internet, (4) ubicuidad de la tecnología, y (5) hábitos de consumo.

\section{Discusión}

\section{Estar presentes a través de mensajes de voz e imágenes}

Tal como lo demuestra la evidencia, para los participantes, el factor económico fue la causa principal de la migración. Esto significa que mantienen responsabilidades económicas desde Chile con sus familiares en Colombia y deben enviar dinero para apoyarlos. Esta situación, hace que sus trabajos sean vitales para la supervivencia de sus familias quienes dependen en gran medida de este ingreso, exponiéndolos a aceptar diferentes situaciones de discriminación o inseguridad laboral que son comunes entre migrantes en Chile. Estas responsabilidades financieras también imponen la urgencia de encontrar diversas fuentes de empleo que aseguren un recurso económico, obligándolas a trabajar en horas que generalmente se dedican al descanso, tal como lo cuentan dos mujeres que dejaron a sus hijos en Colombia:

\footnotetext{
"Se tiene que trabajar un poco más, no sólo se depende de un ingreso, siempre busco formas de obtener salarios adicionales, aquí en Chile hay muchas oportunidades, aunque tenemos que venir a trabajar un domingo (...) Obviamente, también hay personas que se aprovechan y pagan menos al venezolano, al colombiano”. (Ana, 48, vendedora, migrante Chile)

"Tengo un horario fijo que es de 8 a 4 de la tarde. Sin embargo, trato trabajar los domingos porque eso se paga extra. Aquí tenemos que trabajar y no descansar, como dicen”. (Patricia, 47 años, comerciante, migrante Chile)
}

Ambos testimonios hablan de la relevancia que tienen sus compromisos laborales en su experiencia migratoria. Esta realidad económica también ha afectado dramáticamente su enfoque y uso de tecnologías para la comunicación con sus familias. En primer lugar, porque como los ritmos de su trabajo los obligan a estar ocupados durante buena parte del día, la comunicación debe ajustarse a esas rutinas, quedando fragmentadas, imprecisas, pero a la vez constantes, como comenta Patricia:

La mayoría de las llamadas son rápidas, de las que puedo hablar porque tengo que asistir [a la tienda]. Sin embargo, también puedo recibir mensajes o audios. Desde la llamada de voz, escucho y te sientes más cerca porque sabes que la persona está en línea. (Patricia, 47 años, comerciante, migrante Chile) 
Estar en línea es, para Patricia, un sinónimo de sentirse más cerca. Esta posibilidad de comunicación diaria y de diferentes maneras (voz, video-llamadas, textos) que proporcionan aplicaciones como WhatsApp es percibida por los participantes como necesaria para mantener la relación. Reconocen que les hace sentirse como en casa, y "eso hace que las distancias sean más tolerables" (Patricia, 47 años, comerciante, migrante Chile). Otro aspecto relevante es que además de la llamada, ella puede recibir mensajes de voz, otorgando certeza sobre la comunicación constante, aunque sólo puede revisarlos cuando su trabajo se lo permite. Estos son espacios comunicativos no sincrónicos que dan vida a los espacios translocales (Burrell y Anderson, 2008) y esta idea de hibridación en la comunicación, es un espacio que nace y da forma en la flexibilidad que les ofrece el online.

Una de las sorpresas entre los datos recogidos, fue la pérdida de popularidad de la red Facebook. Esta red a la fecha, ha dado cuenta cómo por años ha sido la favorita para compartir fotos y comentarios, convirtiéndose en una especie de bitácora de los migrantes (Pavez, 2014). Skype, que en otros estudios ha sido predominante para encontrar un espacio digital para que la familia se conecte (King-O'Rain, 2015), en este prácticamente no estuvo presente. En cambio, los participantes en este estudio centran sus comunicaciones en la aplicación de WhatsApp, lo que sigue una tendencia en la región -particularmente en Chile- sobre su alto nivel de adopción. Entre sus motivaciones, durante las entrevistas se mencionó su facilidad de uso, particularmente para aquellos con menores habilidades digitales o menos exposición a internet. También se identificó que ofrece una gran variedad de canales enriqueciendo la comunicación a través de audio, mensajes de texto, mensajes de voz, llamadas de vídeo, envío de imágenes y vídeos. Además, debido a su versatilidad y portabilidad en los teléfonos inteligentes, los participantes señalaron que era adaptable a las situaciones cotidianas para continuar realizando otras actividades. Este es el caso de Jackie (35 años, ayudante en negocio, migrante en Chile) quien trabaja como ayudante en un negocio, la situación económica de su familia se vio profundamente afectada por la cesantía, lo que la obligó a viajar a Chile en búsqueda de nuevas oportunidades, dejando atrás a su hermano en Colombia. En su trabajo de más de 10 horas diarias no hay espacio para estar pendiente de su teléfono o respondiendo mensajes, pero eso no le impide estar permanentemente comunicada con Francisco:

[Me comunico con él a través de] audios y llamadas de WhatsApp porque en mi caso estoy haciendo cosas mientras hablo. No tengo tiempo para escribir, no tengo tiempo. Porque si voy a sentarme y escribir todo el día, puedo perder mi trabajo. Tengo que ser responsable. (Jackie, 35 años, ayudante en negocio, migrante en Chile)

Esta riqueza en las propiedades de la comunicación ha sido muy valorada por Cecilia (23 años, Servicios de Limpieza, migrante en Chile), esta joven colombiana que trabaja en una precaria situación en una empresa de limpieza, descubrió que estaba embarazada solamente dos semanas después de haber migrado a Chile. Debido al temor de que su familia se enterara y molestara con ella, decidió seguir adelante con el embarazo y comunicar la noticia tres meses después del nacimiento del bebé. Esto lo hizo con un mensaje de voz de WhatsApp acompañado por una foto de Cristóbal, su hijo. El impacto lo recibió su madre, Magdalena:

"Fue muy duro porque yo no sabía cómo apoyarla estando acá [Cali], por eso quiero ir allá lo más pronto posible, ya estoy viendo pasaje. Fue difícil porque nunca me contó hasta que ya estaba nacido. No sabía qué hacer cuando me lo dijo....”. (Magdalena, 45 años, su hija migró a Chile)

Tanto para Cecilia como para Magdalena la comunicación a través de este chat se ha convertido en un elemento central de su día a día. También con su novio y padre de Cristóbal, quien también está en Colombia:

[Uso mucho] la video-llamada, siento que están aquí conmigo porque los veo [a sus padres]. Los estoy viendo, qué están haciendo, qué pasa entre ellos con el mensaje de voz, yo a menudo expreso lo que siento. Él [el novio] también me responde con una nota de voz, y por eso escucho su voz. Me gusta escucharlo cuando me dice que me ama, que me echa de menos, que si él pudiera enviaría a alguien a venir por mí . (Cecilia, 23 años, Servicios de Limpieza, migrante en Chile)

Estas comunicaciones de voz son claves para Cecilia, particularmente en su contexto de precariedad, alquilando una habitación pequeña, trabajando a tiempo completo y dependiendo de una organización sin 
fines de lucro para cuidar a Cristóbal, que al momento de la entrevista tenía seis meses de edad. Por lo tanto, poder contar con su familia y tener sus palabras de aliento es fundamental para ella. Su familia en el otro extremo, está ansiosa por el bebé y por cómo ella puede trabajar, cuidarlo sola y adecuadamente. Los videos, llamadas y mensajes de voz son tranquilizadores para ambas partes, tal como han descubierto otros investigadores (Peñaranda, 2010).

\section{Nuevos rituales de comunicación}

Otro aspecto común entre los participantes fue su interés por mantener una relación cercana con sus familiares, amigos y seres queridos a pesar de vivir en un país diferente. Entre sus experiencias, estas relaciones se basan en su capacidad de estar continuamente presentes en su vida cotidiana y actividades mediadas por las plataformas de medios sociales. Según lo discutido por Madianou (2014), los participantes destacaron que lo que influyó en su decisión de migrar fue que tienen la oportunidad de permanecer "cerca digitalmente", como lo expresó Francisco (37 años, comerciante, hermano de migrante) un entrevistado. En este sentido, los participantes que viven en Chile y Colombia valoraron mucho los desarrollos tecnológicos que les permitieron mantener conversaciones e intercambiar fotografías y vídeos con regularidad y a un costo relativamente accesible o mediante wifi gratuito en lugares públicos. Es el caso de Nicole (27 años, ayudante de cocina, migrante), que dejó su ciudad natal en Tuluá hace 8 meses y trabaja como ayudante de cocina. La presión para mantener a su madre y su hermano pequeño, y la mala situación económica en su ciudad, motivó su primera experiencia migratoria. Sin embargo, su llegada a Chile no ha estado exenta de problemas, ya que en el momento de la entrevista no había encontrado un trabajo estable. Este tipo de situaciones complejas son los que mantienen a María -su madre- muy preocupada. Además, porque ella ya tiene un hijo que emigró a Brasil por la misma razón. Para esta madre, la conversación diaria a través de WhatsApp, ya sea por texto o llamadas de voz con sus hijos es crucial:

Con la primera persona con la que me comunico por la mañana, es con mi hijo en Brasil. Siempre estoy preocupada por él porque trabaja en la calle, es comerciante. Luego, a mediodía, él me llama y más tarde hablamos un poco por la noche. Nicole, mi hija en Chile, me llama cuando está desocupada, hace videollamadas y hablamos por mucho tiempo: una hora, media hora, tenemos muy buena comunicación todos los días. Hablamos. Le pregunto qué estás haciendo, qué comiste, todas esas pequeñas cosas, cuando tiene que ir a trabajar en horas extrañas, le pido que me marque cuando llegue. (María, 52, su hija migró a Chile)

El caso de María es un ejemplo de algunos de los aspectos que definen su rol como madre y continúan, uno de ellos es el estar preocupada constantemente por el paradero de sus hijos. El rol de la tecnología en los padres es similar a los hallazgos de Katz y González (2016), en particular por el apoyo emocional y la participación en la atención transnacional, brindando sostén a los esfuerzos de los padres por mantener la continuidad familiar. Así como otros autores han discutido este tema sobre la crianza de los hijos (Chib et al., 2014), las llamadas y los mensajes de monitoreo para aliviar los temores, también se encontraron estos comportamientos en madres con hijos adultos. Al igual que ella depende en gran medida de WhatsApp para poder seguir sus pasos y tener la confirmación de que Luisa llegó con seguridad, como ha descubierto la investigación, esto disminuye la ansiedad que produce la separación y proporciona una sensación de cercanía (Peñaranda, 2010). Sin embargo, es una práctica que se perpetúa en los casos analizados y es deseada también por los hijos, pues en vez de calificar estas llamadas como un constante monitoreo, las ven como un espacio de encuentro cotidiano. Este es el caso de Manuela y Elisa con sus respectivas madres, han mantenido una relación cercana gracias a tres videollamadas diarias que ya son parte de su rutina:

Digo que mi madre tiene alas porque sueña y lucha por lograr lo que quiere. (...) Al principio era difícil no tenerla cerca, pero uno se acostumbra (...). En un día normal tenemos tres video-llamadas: por la mañana, para los buenos días, a la mitad del día para ver cómo estoy o si estoy en casa y por la noche el resumen del día. (Manuela, 18 años, su madre migró a Chile). 
Todas las mañanas, cuando me levanto, tengo el saludo de mi mamá en mi teléfono, 'Dios bendiga a mi hija' y respondo 'hola mami, bendiciones, gracias'. Le digo en cuanto salgo del trabajo, cuando llego, hablo a ella constantemente. Por la noche también está la videollamada habitual de WhatsApp . (Elisa, 21 años, limpieza, migrante en Chile)

Ambos testimonios hablan del lado emocional del proceso migratorio. El vínculo madre-hija, por ejemplo, encontró una nueva forma de expresarse mediante la creación de hábitos que les ayudan a continuar adelante, sobre todo en escenarios complejos de aislación y vulnerabilidad como la migración económica, sin otros acompañantes del grupo familiar. Tal y como comentan otros autores (Brüggemann y Wessler, 2014; Nedelcu y Wyss, 2016), se establecen nuevos rituales y escenarios emocionales que permiten la presencia conjunta de los miembros de la familia y proporcionan nuevas formas de intimidad que enriquecen su vida cotidiana. Además, las tres rutinas de video-llamadas mencionadas por Manuela fueron recurrentes entre las mujeres que participaron en el estudio, ya que evidencian gran importancia para poder desear un buen día o dar una bendición matutina al miembro de la familia que está lejos, lo cual también habla de sus raíces latinas y vínculos emocionales (Ramírez, 2014). Estos rituales ayudan a recrear los sentidos de pertenencia que en este caso fueron facilitados por la portabilidad del teléfono inteligente, permitiendo ser acompañados por el otro. La sensación de distancia se alivia con esta presencia virtual y el contacto cotidiano (Tuñón y Mena, 2018).

\section{El sentido del deber emocional}

Otra tendencia de los colombianos migrantes en este proyecto es su sentido del deber que supera el aspecto económico, tocando los deberes emocionales. Además del envío de remesas, deben lidiar con una responsabilidad emocional para continuar con sus roles familiares, lo cual se intensifica con la distancia, particularmente de la persona que permanece en el país de origen. La separación y las oportunidades tecnológicas para permanecer conectados generan una especie de contradicción entre la confirmación de la soledad y también el apoyo creciente del grupo familiar. Esto se encontró anteriormente en otras investigaciones donde se afirmaba que la comunicación regular a veces se intensifica, en lugar de disminuir, debido a la sensación de distancia (Wilding, 2006). Es el caso de las hermanas Rocío (24 años, hermana de migrante) y Francisca (20 años, hermana migró a Chile), a quienes la muerte de su madre hace poco más de un año les ha hecho sentirse más responsables la una de la otra. Aunque pueden comunicarse diariamente, ambas confirmaron que no estar físicamente presente entre ellas, tiene un costo emocional. Además, porque Francisca tuvo que aprender a usar WhatsApp para participar activamente en el grupo familiar para obtener actualizaciones de su hermana, lo cual no es raro en la familia que permanece en el país de origen (Pearce y Rice, 2013). Según Francisca:

“[La relación] ha mejorado porque somos más abiertas, y estamos más conscientes la una de la otro, pero a veces ella tiene problemas, y me encantaría estar allí en el mismo lugar, y hablamos por teléfono, pero no es lo mismo, eso es lo que ha cambiado. Ella pasa por momentos que se siente muy sola, y no puedo estar allí para abrazarla “. (Francisca, 20 años, hermana migró a Chile)

Del relato de Francisca y de otros participantes es posible analizar dos aspectos de las comunicaciones y la migración. Primero, que la riqueza de la tecnología es realmente apreciada para los participantes en ambos extremos, ya que pueden hablar, enviar mensajes y también ver, en algunos casos, tantas veces como lo desee la otra persona. Esto permite la co-presencia con la intención de continuar integrando al miembro de la familia a la rutina en el país de origen de forma de ayudarle a sobrellevar la distancia (Pearce y Rice, 2013). Además, porque es posible encontrar un espacio para el encuentro virtual que brinde apoyo emocional (Bailey 2007; Wilding, 2006). Esta instancia permite esta idea de familia imaginada de la que habla Vermot (2015), que implica ajustarse a nuevos tipos de comunicaciones que inevitablemente cambian las dinámicas familiares, como en este caso, mejorando la comunicación. 
Sin embargo, los participantes también se vuelven dependientes de la tecnología y sus aspectos técnicos, como la calidad de la señal, para tener acceso a wifi o un plan, y además para tener las habilidades digitales para maniobrar. Esta es la situación que enfrenta José (35 años, ayudante de cocina, migrante en Chile), quien vive en Chile desde 2013, dejando atrás a sus padres y hermanos para cumplir su sueño de convertirse en chef. Todo su esfuerzo económico ha consistido en tener un teléfono inteligente de buena calidad y un plan que permita su comunicación constante con Colombia para hacer frente al "dolor, la nostalgia y la angustia" que siente por no poder estar con ellos en momentos específicos, como cuando su madre se rompió la cadera. También tiene muy presente en su mente lo afortunado que es tecnológicamente hablando en comparación con su padre que emigró hace casi tres décadas a los Estados Unidos:

\footnotetext{
"Recuerdo que sí nos llamaba, inicialmente, era una vez al mes, una vez cada 15 días porque las llamadas eran costosas. Ahora es completamente diferente, puedo hablar contigo y puedo recibir una llamada de mi madre de Colombia, una llamada de una tía en los Estados Unidos o una llamada de un amigo en Ámsterdam, así. Es como si uno estuviera allí, estoy mucho más lejos. Sin embargo, el acceso es el mismo" (José, 35 años, ayudante de cocina, migrante en Chile)
}

La tecnología ocupa un lugar esencial en la relación de estas familias. Además, la ubicuidad de los teléfonos inteligentes, que en este caso están habilitados para utilizar principalmente WhatsApp, proporciona a los migrantes la confianza de sentirse cerca, a pesar de que la proximidad física no puede ser reemplazada. Peñaranda (2010) discute esto resaltando la importancia de poder conectarse más allá de las palabras e interpretar lo que no se dice, a través del acceso a la voz e imágenes. Esto fue confirmado por los participantes, que destacaron que un mensaje de texto no les da el nivel de confianza que una comunicación más enriquecida. Por ejemplo, para Fernanda ( 55 años, dueña de casa), la madre de José, esta idea de poder llamar a su hijo sin mayor costo y con la regularidad que ella estime, le da la seguridad que necesita porque le permite "escuchar a mi hijo, de saber realmente cómo está" tal como dice Fernanda ( 55 años, dueña de casa, madre de migrante). Esta riqueza de comunicación también habla de los nuevos códigos espaciales argumentados por Bell y Erdal (2015) donde la familia se adapta a la distancia a través de medios tecnológicos.

\section{Conclusiones}

Los desarrollos tecnológicos y la movilidad digital ofrecen a los migrantes el potencial de controlar cuándo y cómo abordar la distancia, ya sea para estar en contacto instantáneamente y en tiempo real con sus comunidades de origen o para mantener prácticas y tradiciones (Wilding, 2006). En este contexto, las tecnologías de información y comunicación, desempeñan un papel crucial en estas redes transnacionales así como las configuraciones sociales (Vertovec, 2009). En otras palabras, permiten "hacer familia desde lejos" (Peñaranda et al., 2011, p. 27) creando espacios para la mantención de vínculos entre los miembros de familias transnacionales. Basándose en esta premisa, este artículo explora las tensiones y las negociaciones que se generan producto de estas nuevas prácticas y cómo influye en los roles familiares a pesar de no encontrarse en el país de origen. Específicamente, este estudio indaga la forma en que las redes sociales permiten que los migrantes y sus familias transnacionales alcancen la co-presencia (Baldassar, 2016) y las negociaciones que estas implican.

Entre los resultados, se destaca que WhatsApp es la red social más usada por los participantes, debido a la riqueza de la plataforma respecto a la variedad de posibilidades para conectarse de forma síncrona y no síncrona, permitiendo de esta manera la co-presencia en los participantes. Esto lo proporciona el intercambio de mensajes de texto, mensajes de voz, llamadas y video-llamadas. Además, porque era más fácil incorporar esta tecnología en días ocupados y actividades en el día a día de los migrantes. Sin embargo, hay nuevas formas de abordar la vida familiar por el cambio de códigos espaciales y temporales (Bell y Erdal, 2015) donde también se reporta un aumento en la sensación de distancia entre los migrantes y sus familias que el uso de las redes sociales permite a los migrantes enmascarar diariamente. 
La ubicuidad de la tecnología también permitió nuevos rituales de comunicación, discutidos previamente en la literatura para la crianza de niños (Chib et al., 2014), pero en las madres latinas, esto se extendió a los adultos. Sus comunicaciones se basaron principalmente en preocupaciones y ansiedades creadas por distancias al igual que por la vulnerabilidad de las situaciones experimentadas por los participantes, particularmente desde el aspecto económico como la falta de redes sociales de apoyo en Chile. Otro hallazgo fue el sentimiento de responsabilidad emocional: el deber de cuidar al miembro de la familia a distancia y mostrar que pueden estar presentes continuamente, compartiendo temas relevantes, pero cotidianos. Estos también ayudaron a reflexionar sobre el papel de los teléfonos móviles, que están presentes como siempre en la vida de los migrantes y sus familias. Por lo tanto, la necesidad de una comunicación que esté satisfecha con las aplicaciones utilizadas, muestra cómo la tecnología irrumpe en las vidas de las familias cuando uno de sus miembros migra. En este caso de los migrantes colombianos en Chile, en lugar de generar nuevas prácticas familiares, existe un esfuerzo consciente por prolongar en la distancia los roles que ya existen.

Futuros estudios sobre el tema podrían considerar el papel de WhatsApp como una red social emergente que está asumiendo un papel predominante, incluso más allá de Facebook. Además, cómo sus diferentes canales para la comunicación sincrónica y asincrónica tienen un impacto en las relaciones y los roles familiares a distancia. Otro aspecto para considerar, y que no fue parte de este estudio, es cómo el consumo de TIC actúa como una plataforma que les permite situarse digitalmente en su país de origen incide en sus procesos de integración a la sociedad de acogida, puesto que es posible crear una burbuja digital producto de un menor consumo cultural e interacciones con la comunidad de acogida aumentando su marginalización.

\section{Referencias}

Ávalos, B. (2012). Inmigrantes en Chile: Límites y potencialidades de los niveles locales para construir un nuevo pacto de integración social un estudio de caso de la comuna de Santiago. [Tesis pregrado, no publicada] Universidad de Chile

Banco de Desarrollo de América Latina, (2013). Hacia la transformación digital de América Latina: las infraestructuras $y$ los servicios TIC en la región. http://publicaciones.caf.com/media/39809/informe_tecnologiacaf.pdf

Baldassar, L. (2016). De-demonizing distance in mobile family lives: co-presence, care circulation and polymedia as vibrant matter. Global Networks, 16(2), 145-163. https://doi.org/10.1111/glob.12109

Bailey, O, Georgiou, M. y Harindranath, R (Eds.) (2007). Transnational lives and the media:re-imagining diaspora. Palgrave Macmillan.

Bailey, O. (2007). Transnational Identities and the Media. In O. Bailey, M. Georgiou y R. Harindranath (Eds.) Transnational lives and the media: re-imagining diaspora (pp. 212-230). Palgrave Macmillan.

Bhabha, H. (1996). Cultures' In-Between. In S. Hall y P. du Gay (Eds.), Questions of cultural identity (pp. 53-60). SAGE.

Bell, J., y Erdal, M. (2015). Limited but enduring transnational ties? Transnational family life among Polish migrants in Norway. Studia Migracyjne-Przeglad Polonijny, 41(3), 77-98.

Benitez, J. L. (2006). Transnational dimensions of the digital divide among Salvadoran immigrants in the Washington DC metropolitan area. Global Networks, 6(2), 181-199. https://doi.org/10.1111/j.1471-0374.2006.00140.x

Boccagni, P., y Baldassar, L. (2015). Emotions on the move: Mapping the emergent field of emotion and migration. Emotion, Space and Society, 16, 73-80. https://doi.org/10.1016/j.emospa.2015.06.009

Boyatzis, R. E. (1998). Transforming qualitative information: Thematic analysis and code development. S.AGE

Brinkerhoff, J. M. (2009). Digital diasporas: Identity and transnational engagement. Cambridge University Press.

Bryman, A. (2012). Social research methods (4th ed.). Oxford University Press.

Brüggemann, M., y Wessler, H. (2014). Transnational Communication as Deliberation, Ritual, and Strategy. Communication Theory, 24(4), 394-414. https://doi.org/10.1111/comt.12046 
Burrell, J., y Anderson, K. (2008). 'I have great desires to look beyond my world': trajectories of information and communication technology use among Ghanaians living abroad. New Media y Society, 10(2), 203-224. https:/ /doi.org/10.1177/1461444807086472

Cano, V. y Sofia M. (2009) Los estudios sobre migración internacional en Chile: apuntes y comentarios para una agenda de investigación actualizada. Papelesde población, 15(61), 129-167. https://revistes.ub.edu/index.php/b3w/art icle/view/31496/32288

CEPAL \& Organización Internacional del Trabajo. (2017). Coyuntura Laboral en América Latina y el Caribe: la inmigración laboral en América Latina. Naciones Unidas.

Chib, A., Malik, S., Aricat, R. G., y Kadir, S. Z. (2014). Migrant mothering and mobile phones: Negotiations of transnational identity. Mobile Media y Communication, 2(1), 73-93. https://doi.org/10.1177/2050157913506 007

Christiansen, C. (2004). News media consumption among immigrants in Europe: The relevance of diaspora. Ethnicities, 4(2), 185-207. https://doi.org/10.1177/1468796804042603

Contreras, V. R. (2014). The role of drug-related violence and extortion in promoting Mexican migration: Unexpected consequences of a drug war. Latin American Research Review, 49(3), 199-217. https://www.jstor.org/stable/4 3670200

Courtois, C., Mechant, P., Paulussen, S. \& de Marez, L. (2012). The triple articulation of media technologies in teenage media consumption. New Media \& Society, 14(3), 401-420. https://doi.org/10.1177/1461444811415046

Dekker, R., Engbersen, G., y Faber, M. (2016). The use of online media in migration networks. Population, Space and Place, 22(6), 539-551. https://doi.org/10.1002/psp.1938

Departamento de Extranjería y Migración. [DEM] (s.f.) Estadisticas Migratorias. Ministerio del Interior y Seguridad Pública. http://www.extranjeria.gob.cl/estadisticas-migratorias

Diminescu, D. (2008). The connected migrant: an epistemological manifesto. Social Science Information, 47(4), 565-579. https://doi.org/10.1177/0539018408096447

Doveling, K., Harju, A., y Sommer, D. (2018). From mediatized Emotion to Digital Affect Cultures: New Technologies and Global. Flows of Emotion. Social Media + Society 4(1), 1-11. https://doi.org/10.1177/2056305117743141

Esterberg, K. G. (2002). Qualitative methods in social research. Mc Graw Hill.

Feng, Y.y Ekiaka, V. (2011). Impact of Spanish-Speaking Media technologies on Latino University Students' Bicultural Identity Development. Journal of Border Educational Research, 10(1), 192-214. https://journals.tdl.org/jber/in dex.php/jber/article/view/7206/6449

Fielding, N. G., y Thomas, H. (2008). Qualitative interviewing. In Gilbert, N. Researching Social Life (pp. 123-144). Sage.

Francisco, V. (2015). 'The Internet Is Magic': Technology, Intimacy and Transnational Families. Critical Sociology, 41(1), 173-190. https://doi.org/10.1177/0896920513484602

Gaskell, G. (2000). Individual and group interviewing. In M. Bauer y G. Gaskell, (Eds.). Qualitative researching with text, image and sound: A practical handbook fsocial researchrch (pp. 38-56). Sage.

Georgiou, M. (2005). Mapping diasporic media cultures: a transnational cultural approach to exclusion. In R. Silverstone (Ed.). Media, technology and everyday life in Europe: from information to communication (pp. 33-52). Ashgate.

Georgiou, M. (2006).Diaspora, identity and the media: diasporic transnationalism and mediated spacialities. Hampton Press.

Georgiou, M. (2013). Seeking Ontological Security beyond the Nation. The Role of Transnational Television. Television and New Media, 14(4), 304-321. https://doi.org/10.1177/1527476412463448

Gillespie, M. (1995). Television, ethnicity and cultural change. London: Routledge

Giddens, A. (1990). The Consequences of Modernity. Stanford University Press.

Greschke, M. (2012). Is there a home in cyberspace: the Internet in migrants' everyday life and the emergence of global communities. Routledge. 
Katz, V. S., y Gonzalez, C. (2016). Community variations in low-income Latino families' technology adoption and integration. American Behavioral Scientist, 60(1), 59-80. https://doi.org/10.1177\%2F0002764215601712

King-O'Riain, R. C. (2014). Transconnective space, emotions, and Skype. En T. Benski y E. Fisher (Eds) Internet and emotions (pp. 131-43). Routledge.

Hiller, H., y Franz, T. (2004). New ties, old ties and lost ties: the use of the internet in diaspora. New media y Society, 6(6), 731-752. https://doi.org/10.1177/146144804044327

Hootsuite (2018). Digital in 2018: The Americas. https://hootsuite.com/resources/digital-in-2018-americas

Hartmann, M. (2006). The triple articulation of ICTs. Media as technological objects, symbolic environments and individual texts. En T. Berker, M. Hartmann, Y. Punie \& K. Ward (Eds.), Domestication of media and technology (pp.80-102). Maidenhead: Open University Press.

Karim, K. H. (1998). From ethnic media to global media: Transnational communication networks among diasporic communities. University of Oxford. Transnational Communities Programme.

Kim, H., Monk, A., Wood, G., Blythe, M., Wallace, J., \& Olivier, P. (2013). TimelyPresent: Connecting families across continents. International Journal of Human-Computer Studies, 71(10), 1003-1011. https://doi.org/10.1016/j. ijhcs.2013.05.001

King-O’Riain, R. (2015) Emotional streaming and transconnectivity: Skype and emotion practices in transnational families in Ireland. Global Networks, 15(2), 256-273. https://doi.org/10.1111/glob.12072

de Leeuw, S., \& Rydin, I. (2007). Diasporic Mediated Spaces. En O. Bailey, M. Georgiou \& R. Harindranath (Eds.), Transnational lives and the media: re-imagining diasporas (pp. 175-194). Pallgrave.

Libertad y Desarrollo (2019). Encuesta Casen 2017: Radiografía a la inmigración en Chile. Temas Públicos 1384 (1)

Lutz, H. (2011). The new maids: transnational women and the care economy. Zed Books.

Madianou, M. (2014). Smartphones as polymedia. Journal of Computer-Mediated Communication, 19(3), 667-680. h ttps://doi.org/10.1111/jcc4.12069

Madianou, M. (2016). Ambient co-presence: transnational family practices in polymedia environments. Global Networks, 16(2), 183-201. https://doi.org/10.1111/glob.12105

Madianou, M. y Miller, D. (2012). Migration and New Media: Transnational Families and Polymedia. Routledge.

Martínez, J. y Orrego, C. (2016) Nuevas tendencias y dinámicas migratorias en América Latina y el Caribe. Serie Población y Desarrollo (114). https://repositorio.cepal.org/bitstream/handle/11362/39994/S1600176_es.pdf? sequence $=1 \&$ is Allowed $=\mathrm{y}$

Méndez, L., \& Cárdenas, M. (2012). Hacia la construcción de un modelo comprensivo de análisis de la" situación de inmigración" de mujeres sudamericanas en Chile. Psicoperspectivas, 11(1), 252-272. https://doi.org/10.5027/ps icoperspectivas-Vol11-Issue1-fulltext-192

Miller, D., y Slater, D. (2000). The internet: an ethnographic approach. Berg.

Morgan, D. L., Ataie, J., Carder, P., y Hoffman, K. (2013). Introducing dyadic interviews as a method for collecting qualitative data. Qualitative health research, 23(9), 1276-1284. https://doi.org/10.1177/1049732313501889

Morgan, D. L., Eliot, S., Lowe, R. A., y Gorman, P. (2016). Dyadic interviews as a tool for qualitative evaluation. American Journal of Evaluation, 37(1), 109-117. https://doi.org/10.1177/1098214015611244

Nedelcu, M., y Wyss, M. (2016). 'Doing family' through ICT mediated ordinary co-presence: transnational communication practices of Romanian migrants in Switzerland. Global Networks, 16(2), 202-218. https://doi .org/10.1111/glob.12110

Pavez, I. (2014). The Latinas' Internet: Meanings and practices in the everyday lives of disadvantaged migrant women in London [Doctoral theses, London School of Economics and Political Science). LSE Theses Online. http:// etheses.lse.ac.uk/982/

Pearce, K. y Rice, R. (2013). Digital divides from access to activities: Comparing mobile and personal computer Internet users. Journal of Communication, 63(4), 721-744. https://doi.org/10.1111/jcom.12045 
Peñaranda, M.C., Vitores, A., Martínez, L. M., Muñoz Justicia, J., y Íñiguez-Rueda, L. (2011). El acceso público a las tecnologías de la información y la comunicación: el lugar de los locutorios en los procesos migratorios. Papeles del CEIC, 70(1), 1-37. https://identidadcolectiva.es/pdf/70.pdf

Peñaranda, M.C. (2010). “Te escuchas aquí al lado”: Usos de las tecnologías de la información y la comunicación en contextos migratorios transnacionales. Atheneadigital: revista de pensamiento e investigación social, (19), 0239-248. https://ddd.uab.cat/pub/athdig/15788946n19/15788946n19p239.pdf

Ramírez, J. P. (2014). Uso de tecnologías de la información y la comunicación en familias caleñas con migrantes en España. Revista de Estudios Sociales, 48, 110-123. https://doi.org/10.7440/res48.2014.09

Rodríguez, L. (2011). Siempre estamos conectados: así sé que me quiere mucho. Comunicaciones en familias transnacionales a través de internet. RevistaLatinoamericana de Estudios de Familia, 3, 50-64. http://biblioteca. clacso.edu.ar/gsdl/collect/co/co-035/index/assoc/D14048.dir/RLEF3_Completa.pdf\#page $=51$

Rojas, N. y Silva, C. (2016) La migración en Chile: Breve reporte y caracterización [Migration in Chile: Brief report and characterization]. Observatorio Iberoamericano sobre Movilidad Humana. Migración y Desarrollo, OBIMID.

Schiller, N., Basch, L., y Szanton Blanc, C. (1995). From Immigrant to Transmigrant: Theorizing Transnational Migration. Anthropological Quarterly, 68(1), 48-63. https://doi.org/10.2307/3317464

Silva, J. y Lufin, M. (2013). Situación de inmigración y asentamiento de grupos Afrocolombianos en la Región Antofagasta, con énfasis en las mujeres. Documentos de Trabajo en Economia y Ciencia Regional 35, Universidad Católica del Norte, Chile. https://9f7dc88f-a-ce2465ef-s-sites.googlegroups.com/a/ucn.cl/wpeconomia/archivos/WP2013-04.pd f?attachauth=ANoY7crR4nOjE_lJLElalFkBrSWpNchwE3GmO9t-Pbuo8adf8PYgszp7hnhJd59JG1UER4Z AzuB0Posrf6A9-tI4Ne8nQrNiZTWYydIixEAHrLMfYr_3oGzxKqloDJQeUtvfKehjt1B2dL-DvkNkLfCvJ VdkeVl3EQ2Jdy6V0L5BM9zApbEghkTupDA_2YPtgsnBPuLqbtoK47YPZyzNfpy05k-Tkls_sw\%3D\%3D \&attredirects $=0$

Silva, J., Urzúa, A., Caqueo-Urizar, A., Lufin, M., \& Irarrazaval, M. (2016). Bienestar psicológico y estrategias de aculturación en inmigrantes afrocolombianos en el norte de Chile. Interciencia, 41(12), 804-811.

Taylor, J. (2014). ¿La unión que nos separa? La estructura de la comunidad colombiana en Antofagasta, Chile [The union that separates us? The structure of the Colombian community in Antofagasta, Chile] Master's thesis. Leiden University.

Thayer, L., Córdova, G., y Ávalos, B. (2013). Los límites del reconocimiento: migrantes latinoamericanos en la Región Metropolitana de Santiago de Chile. [The limits of recognition: Latin American migrants in the Metropolitan Region of Santiago de Chile] Perfiles Latinoamericanos, 21(42), 163-191. https://doi.org/10.18504/PL2142163-2013

Torres, J. V., y Aguilar, L. J. (2018). Tendencias, Oportunidades y Retos del uso de las Redes Sociales en Latinoamérica: Caso Centroamérica y Panamá. KnE Engineering, 3(2), 931- 941. https://doi.org/10.18502/keg.v3i1.1513

Toral, E. (2017). Tecnologías de información y comunicación y migración en el nuevo espacio audiovisual : estudio de cuatro comunidades del sur del Ecuador. En A. Escudero \& D. González (Eds.) Escenariosy desafíos de la comunicación y la cultura en el espacio audiovisual iberoamericano(pp. 214-242). Sevilla, Universidad Internacional de Andalucía. http://hdl.handle.net/10334/3832

Tuñón, E., \& Mena, R. (2018). Familias transnacionales y prácticas sociodigitales en Nueva York. Norteamérica, 13(1), 33-55. https://doi.org/10.20999/nam.2018.a002

Verdery, A., Mouw, T., Edelblute, H., y Chavez, S. (2017). Communication flows and the durability of a transnational social field. Social Networks 53, 57-71. https://doi.org/10.1016/j.socnet.2017.03.002

Vertovec, S. (2009). Transnationalism. Routledge.

Vermot, C. (2015). Guilt: A gendered bond within the transnational family. Emotion, Space and Society, 16, 138-146. https://doi.org/10.1016/j.emospa.2015.04.001

Wilding, R. (2006). 'Virtual' intimacies? Families communicating across transnational contexts. Globalnetworks, 6(2), 125-142. https://doi.org/10.1111/j.1471-0374.2006.00137.x 
Witteborn, S. (2012). Forced migrants, new media practices, and the creation of locality. In I. Volkmer (ed.), The handbook of global media research, (pp. 312-330). Wiley-Blackwell

Yañez, S., y Cárdenas, M. (2010). Estrategias de aculturación, indicadores de salud mental y bienestar psicológico en un grupo de inmigrantes sudamericanos en Chile. Saludy Sociedad, 1(1), 51-70. https://www.redalyc.org/pdf /4397/439742462006.pdf

\section{Notas}

* Artículo de investigación científica. Este artículo es resultado de la investigación "Uso de tecnologías de la información y la comunicación en familias colombianas migrantes en Chile" del taller de investigación del Doctorado en Comunicación de la Universidad de los Andes.

1 Nombre de los participantes fue cambiado para respetar su anonimidad.

\section{Licencia Creative Commons CC BY 4.0}

Cómo citar este artículo: Pavez Andonaegui, I., Claro, C., y Burgos Suárez, J. A. (2020). El uso de redes sociales en migrantes Colombianos en Chile. Signo y Pensamiento, 39(76). https://doi.org/10.11144/Javeriana.syp 39-76.ursm 